Revue Interventions économiques

Papers in Political Economy

\title{
Théorie de l'entreprise sociale et pluralisme : L'entreprise sociale de type solidaire
}

Social Enterprise Theory and Pluralism: Social Enterprise of the Solidarity Type

Jean Louis Laville, Isabelle Hillenkamp, Philippe Eynaud, Jose Luis Coraggio, Adriane Ferrarini, Genauto Carvalho de França Filho, Luis Inácio Gaiger, Kenichi Kitajima, Andrea Lemaître, Youssef Sadik, Marilia Veronese et Fernanda Wanderley

\section{OpenEdition}

Journals

Édition électronique

URL : http://journals.openedition.org/interventionseconomiques/2771

DOI : 10.4000/interventionseconomiques.2771

ISBN : $1710-7377$

ISSN : $1710-7377$

Éditeur

Association d'Économie Politique

Référence électronique

Jean Louis Laville, Isabelle Hillenkamp, Philippe Eynaud, Jose Luis Coraggio, Adriane Ferrarini, Genauto Carvalho de França Filho, Luis Inácio Gaiger, Kenichi Kitajima, Andrea Lemaître, Youssef Sadik, Marilia Veronese et Fernanda Wanderley, « Théorie de l'entreprise sociale et pluralisme L'entreprise sociale de type solidaire », Revue Interventions économiques [En ligne], 54 | 2016, mis en ligne le 01 mars 2016, consulté le 14 juin 2019. URL : http://journals.openedition.org/ interventionseconomiques/2771; DOI : 10.4000/interventionseconomiques.2771

Ce document a été généré automatiquement le 14 juin 2019.

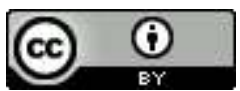

Les contenus de la revue Interventions économiques sont mis à disposition selon les termes de la Licence Creative Commons Attribution 4.0 International. 


\title{
Théorie de l'entreprise sociale et pluralisme : L'entreprise sociale de type solidaire
}

\author{
Social Enterprise Theory and Pluralism: Social Enterprise of the Solidarity Type \\ Jean Louis Laville, Isabelle Hillenkamp, Philippe Eynaud, Jose Luis \\ Coraggio, Adriane Ferrarini, Genauto Carvalho de França Filho, Luis \\ Inácio Gaiger, Kenichi Kitajima, Andrea Lemaître, Youssef Sadik, Marilia \\ Veronese et Fernanda Wanderley
}

\section{Introduction}

1 À partir de recherches menées dans les années 1990, le réseau de recherche européenne EMES $^{1}$ a défini un idéal-type d'entreprise sociale ${ }^{2}$ qui se positionne par rapport aux courants de pensée nord-américains sur l'entreprise sociale. Il identifie neuf dimensions caractéristiques des entreprises sociales, dans les domaines de l'économie, du social et de la gouvernance.

2 Il apparait que cet idéal-type d'EMES, construit à partir de données européennes datant de la fin du $\mathrm{XX}^{\mathrm{e}}$ siècle peut être questionné à partir de l'élargissement des formes d'entreprise sociale en Europe en ce début de XXI ${ }^{e}$ siècle marqué par la crise; et aussi à partir de modèles d'entreprises sociales présents dans d'autres parties du monde et intégrant la solidarité comme logique et valeur fondamentale dans des actions collectives à dimension non seulement socioéconomique, mais aussi sociopolitique. C'est pourquoi il s'avère important d'élaborer un idéal-type d'entreprise sociale dans une perspective d'économie solidaire (ou entreprise solidaire).

Ce texte propose à la discussion une élaboration préliminaire de cet idéal-type à partir d'un ensemble, non exhaustif, de recherches identifiées dans le champ de l'économie solidaire. Après avoir précisé l'apport et les limites des approches existantes de l'entreprise sociale, il situe un certain nombre d'expériences d'entreprise solidaire puis propose neuf indicateurs de cet idéal-type, en référence à l'idéal-type d'EMES. Il aboutit à 
préciser certains indicateurs (dimensions économiques et sociales) et à en proposer de nouveaux (dimension politique). Le résultat est un idéal-type de l'entreprise solidaire en dialogue avec celui de l'entreprise sociale, pouvant jouer un rôle complémentaire permettant de préciser les contours de ce dernier, ce qui est sans doute indispensable comme point de départ pour identifier la pluralité de modèles d'entreprise sociale existant au niveau international.

\section{Les approches de l'entreprise sociale}

4 Les approches de l'entreprise sociale ont ceci d'important par rapport aux approches dominantes en économie de l'entreprise et sciences de gestion qu'elles ont permis d'interroger les finalités de l'entreprise et d'une partie de l'action économique. Quels que soient les sensibilités et courants de pensée au sein de ces approches, elles s'accordent à mettre en avant une finalité sociale de l'entreprise, qu'il s'agisse de réinsérer des chômeurs dans le marché du travail, de proposer de nouveaux services de proximité, de revitaliser des territoires à partir de l'entraide entre leurs habitants, ou encore de promouvoir des démarches de développement local durable. Ces approches constituent donc une avancée par rapport à celles qui se focalisent sur la maximisation du profit au travers de l'objectif de création de valeur pour l'actionnaire.

5 Au sein de ces approches existent des courants qui se caractérisent par certaines spécificités et qui correspondent à des contributions respectivement nord-américaines et européennes.

\section{Des contributions nord-américaines et européennes}

6 Les courants nord-américains se positionnent plutôt par rapport au marché et à l'innovation sociale. Un premier courant, représenté notamment par James Austin et ses collègues (2006) de la Harvard Business School, met l'accent sur le recours à des ressources marchandes comme moyen pour des associations à but non lucratif de réaliser leur mission sociale. Le second, inspiré par la figure de l'entrepreneur innovant susceptible de répondre à des besoins sociaux, envisage d'emblée que différents types d'entreprises puissent contribuer à un objectif social, qu'elles soient à but lucratif ou non (Dees 1998 ; Salamon et Young, 2002). On peut penser que sous l'effet du rapprochement entre ces deux courants, l'entreprise sociale outre-Atlantique est aujourd'hui abordée au travers de sa mission sociale pour la réalisation de laquelle l'augmentation des ressources marchandes est préconisée, sans nécessaire rapport avec le type d'activité économique ni avec la structure de gouvernance. Le critère de non-distribution des profits qui était central pour le tiers secteur a, en particulier, été progressivement relativisé, voire abandonné pour l'entreprise sociale. Quant aux dynamiques d'innovation sociale, elles sont appréhendées surtout à travers la valorisation des figures individuelles d'entrepreneurs sociaux et sont considérées comme un moyen de créer une «valeur sociale ». De ce point de vue, les entreprises, la société civile et le secteur public sont considérés comme complémentaires et la distinction, entre entreprises sociales sur le marché et celles créant des équilibres économiques en mobilisant aussi des ressources non marchandes, n'est pas essentielle (Ferrarini 2013).

En comparaison, la contribution européenne émanant du réseau des chercheurs d'EMES a pour originalité de combiner la finalité sociale de l'entreprise avec sa structure de 
gouvernance interne. La trajectoire historique des entreprises sociales européennes, qui les lie aux organisations de l'économie sociale, explique ainsi l'importance des critères de participation, de modalités de décision non liées à la détention du capital et de limites à la distribution des profits.

Il existe donc des points communs à l'ensemble de ces courants portant sur la finalité sociale, l'autonomie et la prise de risque économique. Mais, de la conception différente du lien entre mission sociale et structure de gouvernance interne découlent deux différences de taille entre courants nord-américains et européens. Premièrement, dans la contribution européenne, l'importance octroyée à des modes de gestion plus démocratiques crée une distance par rapport aux modes de gestion du secteur privé à but lucratif. Deuxièmement, l'attention portée à des critères de démocratie économique interne dans l'approche européenne permet d'envisager les entreprises sociales comme des partenaires légitimes des politiques publiques, possédant un certain degré d'interaction avec leur environnement institutionnel, ce qui constitue un canal de diffusion pour les innovations sociales qu'elles recèlent. Par contraste, aux États-Unis, la diffusion de l'innovation sociale est considérée comme étant le fruit de l'expansion ou de la multiplication des entreprises sociales, grâce à l'utilisation des ressources marchandes, au soutien de fondations et au dynamisme des entrepreneurs (Defourny et Nyssens 2013).

\section{Les limites des approches existantes de l'entreprise sociale}

Ces approches considèrent dans l'ensemble l'entreprise sociale comme une organisation privée. Pourtant, l'entreprise sociale se situe bien entre sphères privée et publique, au sens où elle peut contribuer à la définition de problèmes publics qui deviennent des objets de débat. Cette dimension publique, au sens de Hannah Arendt (1983) ou de Jürgen Habermas (1988), doit être prise en compte. Pour cela, il convient de suivre ces auteurs dans leur définition du public, tout en se démarquant par ailleurs de la séparation trop stricte qu'ils opèrent entre sphères politique et économique.

Dans cette perspective, il apparait clairement que les activités économiques de l'entreprise solidaire sont indissociables de la dimension institutionnelle, entendue comme confrontation à la question du sens et de la légitimité. Cette dimension concerne deux registres: d'abord celui des logiques instituantes à travers lesquelles les acteurs créent et consolident l'action menée par l'émission de règles, manifestant ainsi leur capacité à générer des communs (Ostrom, 2005) et à exprimer leur volonté transformatrice; ensuite celui du cadre institutionnel, ensemble de normes déjà établies à différentes échelles et dans différents registres, inscrits dans la loi ou non, qui influent sur leur action et sur lequel les entreprises sociales peuvent avoir une certaine influence. L'approche d'EMES a déjà rendu compte des cadres institutionnels dans lesquels évoluent les entreprises sociales et de la manière dont elles peuvent elles-mêmes les influencer. Cependant, le cadre institutionnel y est souvent traité comme un "environnement", séparément du sens et des règles que se donnent les entreprises sociales de manière interne, lesquelles, implicitement, sont considérées comme des organisations privées. Il s'agit ici de contribuer à une analyse institutionnelle plus intégrale et plus critique des entreprises sociales qui tienne compte de leur position à la frontière entre sphères privée et publique.

11 À travers les logiques instituantes, il s'agit notamment de problématiser la question de la mission des entreprises sociales. En se centrant sur les catégories d'« entreprise » 
(définie par rapport à l'activité « économique ») et de « social », ainsi que sur les rapports entre les deux catégories, les approches de l'entreprise sociale risquent d'éviter une réflexion critique sur leur mission et sur les processus de création de valeur sociale. La possibilité d'une interaction positive entre sphère économique et sphère sociale est généralement affirmée sans être interrogée. Le « social » est défini comme une catégorie par défaut au travers de « besoins » non satisfaits par l'Etat et le marché. Le choix d'une mission au sein de ce vaste ensemble peut être effectué par un entrepreneur social ou d'autres décideurs de l'entreprise. La réflexion n'est pas centrée sur la nature et la légitimité des acteurs, sur leur projet politique ni sur les rapports sociaux dans lesquels ils s'inscrivent. Les entreprises sociales prétendent contribuer à une mission sociale en créant une valeur sociale, mais le rapport entre ces entreprises et le débat public est éludé. Certes la définition européenne inclut le fait que l'initiative vienne d'un groupe de citoyens, mais en limitant le fonctionnement démocratique à une égalité formelle entre les membres garantie dans les statuts et sans expliciter les modalités à travers lesquelles se concrétisent des rapports égalitaires.

En négligeant la dimension instituante interne des organisations, cette approche risque entre autres de réduire les différentes formes d'institutionnalisation des entreprises sociales aux formes légales existantes - les coopératives, associations, mutuelles auxquelles s'ajoutent de nouvelles formes spécifiques d'entreprises sociales reconnues dans les législations de certains pays. Dans ce cadre, les entreprises sociales informelles, qui ne s'inscrivent dans aucune législation, sont négligées. Elles tendent à être vues comme une simple « variation » des modalités de référence (Fonteneau et al., 2011: 2 cité dans Gaiger 2013 : 10), alors que dans de nombreux contextes, elles représentent l'un des cas les plus importants numériquement (Gaiger 2013). De plus, on peut penser que les entreprises sociales n'ont pas nécessairement vocation à se formaliser car elles possèdent des logiques instituantes propres à travers leurs règles internes de gestion (ibid.) se manifestant dans leur capacité d'auto-organisation (Veronese, communication personnelle). En complétant ainsi l'analyse organisationnelle par une approche institutionnelle qui ne se limite pas aux cadres légaux existants, il est possible de restituer le sens et les logiques d'ensemble qui se cristallisent dans des formes organisationnelles particulières, légalement reconnues ou non. Si l'on ne considère pas les logiques instituantes qui peuvent se situer dans l'économie informelle ou formelle, alors l'analyse des entreprises sociales court le risque de se convertir en une composante de l'approche néo-modernisatrice, en supposant implicitement une tendance à la formalisation des entreprises sociales informelles.

13 Enfin, l'approche n'est pas centrée sur les rapports entre sphères économique et politique. Elle développe une vision différente de l'entreprise, mais ne met pas radicalement en cause le cadre institutionnel dans lequel l'activité de l'entreprise «se déroule ». Elle admet une capacité « d'agency » des entreprises sociales, mais elle suppose une partition entre trois sphères d'activité - l'économique, le social et le politique - dont les frontières et les interactions ne sont que partiellement interrogées. Questionner les catégories de l'entreprise et du social depuis la perspective de la place de l'économie dans la société, du débat public et de la démocratie apparait donc comme un axe de recherche complémentaire pour l'entreprise sociale. 


\section{Les entreprises sociales de type solidaire}

14 Cette préoccupation théorique entre en résonnance avec des constats empiriques: il existe dans différents continents des initiatives qui peuvent être considérées comme des entreprises sociales et qui ont été créées dans une volonté de transformation des sphères économique et politique. Elles émanent d'une réaction contre l'ordre économique dominant et d'une conscience de la part des acteurs qu' « entre capitalisme et démocratie il y a un rapport de tension insurmontable » (Habermas, 1998, p. 379). En Europe, après la période des Trente Glorieuses (1945-1975) pendant laquelle la perception de cette tension était atténuée, son acuité s'est à nouveau révélée avec l'apparition de nouveaux mouvements sociaux, creusets d'idées alternatives, et dès les années 1970 de pratiques solidaires. Ces expériences voulant par exemple lutter en faveur de l'environnement ou contre le patriarcat ont exigé de « déborder le champ habituel de la démocratie, c'est-àdire le politique, pour investir le domaine économique » (Sousa Santos, Rodriguez, 2013, p. 141). Puis dans les années 1980, elles ont été bousculées par la vague de dérégulations et de flexibilisations préconisée par le consensus de Washington, qui a induit des actions plus défensives de reprises d'entreprises par leurs travailleurs (Paton, 1989) ou d'insertion par l'économique (Gardin, Laville, Nyssens, 2012). En Amérique latine, ces expériences naissent de l'incapacité ou du refus de s'adapter aux conditions du capitalisme périphérique et aux formes de sociabilité qu'il entraine (Gaiger, 2013). Elles se déroulent généralement dans un contexte de précarité sociale et elles visent d'abord à créer des revenus indispensables pour subsister. Mais en même temps, elles réactivent des liens de solidarité, fondés sur des communautés ou associations anciennes ou nouvelles dans les milieux populaires. Les entreprises solidaires opèrent ainsi une inflexion vers des mobilisations plus fortes de la solidarité et de la coopération avec le passage de tactiques de survie à des stratégies (De Certeau, 1980) d'entreprises solidaires. Luis Razeto (1993, p. 40) va jusqu'à avancer que la coopération et la collaboration, qu'il nomme le facteur $\mathrm{C}$, permettent « des économies d'échelle, des économies d'association et des externalités collectives dans le cours de l'action collective » ce qui conduit à redéfinir les notions d'efficacité et d'efficience. De nombreux exemples peuvent être cités.

15 - En Europe différents types d'initiatives pouvant être rapportées aux entreprises sociales affirment leur dimension politique. En Italie du Sud, des coopératives, et en France, les régies de quartier, ont impulsé des dynamiques participatives en liaison avec des autorités publiques locales; au Portugal, le réseau de développement local Animar encourage la démocratisation de l'économie grâce à des expériences territorialisées. En Amérique latine, les entreprises sociales inspirées des approches nord-américaines (par exemple le Social Enterprise Knowledge Network, lié à la Harvard Business School) n'occupent qu'une place mineure dans le débat public et politique. Par contre, les réseaux d'entreprises populaires solidaires, qui lient organisation démocratique des pratiques économiques et positionnement dans l'espace public (França Filho, 2006), jouent un rôle dans le changement institutionnel et la transformation sociale. En particulier au Brésil, en Bolivie, en Équateur et au Nicaragua, ces entreprises ont suscité de nouvelles politiques publiques ou de nouveaux cadres normatifs ou législatifs qui tentent de redéfinir le sens de la modernité à travers une vision plurielle de l'économie (Wanderley, 2009), laquelle n'est bien sûr pas exempte de contradictions ni de dérives. En Afrique du Sud, un nouveau mouvement dit « d'économie solidaire » regroupant différents types de coopératives s'est conformé pour impulser des politiques transformatrices 
(transformative policies), jugeant insuffisants l'économie sociale traditionnelle et la politique actuelle du Black Economic Empowerment (COPAC, 2011).

Afin de ne pas introduire de biais méthodologique en référant la réalité des entreprises sociales uniquement à certains cadres d'analyse proposés au Nord, l'objet de ce texte est de s'appuyer tant sur les exigences conceptuelles ci-dessus que sur des pratiques comme celles qui viennent d'être mentionnées.

\section{Critères de définition de l'entreprise solidaire}

17 Dans cette seconde partie, l'idéal-type est construit selon une méthodologie comparable à celle adoptée pour la définition d'EMES, en étant axé sur l'identification de critères. Ils représentent des indicateurs de différentes dimensions constitutives de l'idéal-type de l'entreprise solidaire comme construction abstraite, et non des propriétés que chaque entreprise solidaire singulière devrait posséder. Par ailleurs, ces critères sont classés selon qu'ils se rapportent à la dimension économique, sociale ou politique des entreprises. Il convient de signaler que ces différentes dimensions s'articulent et se recoupent en pratique. Cette distinction est donc seulement analytique. Les indicateurs économiques visent à caractériser l'organisation de la production et des échanges et les relations de travail dans l'entreprise solidaire idéale typique. Les indicateurs sociaux portent sur le sens de l'action et sur le type de relations sociales, tant internes qu'externes à l'entreprise. Les indicateurs politiques rendent compte de la dynamique instituante des entreprises solidaires et de leur participation à la sphère publique. Cette troisième dimension va donc au-delà des critères de gouvernance dans l'idéal-type d'EMES, qui sont axés sur le mode d'organisation interne des entreprises. L'idéal-type d'entreprise sociale dans une perspective d'économie solidaire dialogue donc avec la définition d'EMES, tout en suggérant de préciser certains critères en référence à des réalités diversifiées.

\section{Indicateurs économiques}

\section{Hybridation des principes économiques et logique de solidarité}

18 Afin de distinguer les entreprises sociales d'organisations à but non lucratif visant uniquement la défense d'intérêts ou l'attribution de ressources (comme les fondations), l'idéal-type d'EMES de l'entreprise sociale définit comme indicateur la production continue de biens ou de services. Par contre, cet indicateur ne distingue pas les entreprises dont l'activité de production de biens ou de services obéit à une logique uniquement marchande, de celles faisant intervenir d'autres principes. Ce choix, qui permet d'inclure les entreprises sociales fondées sur le modèle nord-américain de mobilisation des ressources marchandes, tend à occulter une originalité essentielle des entreprises solidaires qui est la pluralité des principes économiques et l'existence d'une logique de solidarité.

19 Les investigations menées sur l'économie convergent en effet pour réfuter une rationalité économique qui se réduirait à l'intérêt matériel individuel et dont la coordination résulterait uniquement des mécanismes marchands. En accord avec Fernand Braudel (1985, p. 45), qui suggère de ne pas être obnubilé par l'économie de marché « alors qu'elle n'est qu'un fragment d'un vaste ensemble », il importe de reconnaitre avec Karl Polanyi 
(2011) la pluralité des ressources marchandes, mais aussi non marchandes et non monétaires. Aux ressources tirées du marché s'ajoutent celles venues de la redistribution (prélèvement par une autorité centrale et affectation à partir des règles édictées par celle-ci), de la réciprocité (groupements symétriques dont les membres pratiquent une forme de mutualisme) et du partage domestique (production pour l'usage au sein de l'unité domestique selon des règles équitables ou non). Ces principes d'intégration économique peuvent d'ailleurs ne pas être assimilés à de simples ressources et être compris comme des types d'interdépendance, constituant l'élément fondamental de l'analyse de l'économie comme processus institué au sens de Polanyi. La réciprocité ne se réfère alors pas seulement à des ressources mises à disposition, mais à des interdépendances traduisant une complémentarité instituée. La redistribution correspond à des interdépendances établies à travers des systèmes centralisés. Le partage domestiquedécrit les interdépendances changeantes au sein d'unités de type domestique fondées sur l'autosuffisance (Hillenkamp, 2013; Servet 2013). Le marché, enfin, correspond aux interdépendances qui naissent automatiquement entre acheteurs et vendeurs au travers de la fluctuation des prix.

20 Cette interprétation replace les principes de Polanyi dans une vision d'économie politique. Elle engage à interroger les cadres institutionnels et politiques dans lesquels s'inscrivent les entreprises sociales lorsqu'elles mobilisent des ressources de différente nature. Chacun des principes est en effet ambigu. Le principe de réciprocité, en particulier, n'est pas une catégorie automatiquement positive du point de vue de la démocratisation. Comme le partage domestique dans la famille patriarcale, il peut être mobilisé de manière coercitive, par exemple dans des structures de type communautaire. Ce n'est que lorsque la réciprocité est volontairement instituée, dans des structures que les acteurs peuvent d'ailleurs considérer comme modernes ou comme traditionnelles, qu'elle peut prendre un caractère égalitaire et constituer la base de processus de démocratie participative ou délibérative. De même, la redistribution au travers du prélèvement et de la réaffectation des ressources peut être administrée de manière autoritaire, comme elle peut être associée à des modalités de démocratie représentative, $\mathrm{y}$ compris à un niveau local.

21 Dans cette logique, l'entreprise solidaire tend à substituer au principe d'autosuffisance dominant dans l'économie populaire une réciprocité volontairement instituée sur une base d'égalité. Ce type d'entreprise sociale réalise une hybridation entre principes économiques destinée à fournir des moyens pertinents au service de son projet sous l'égide de la réciprocité égalitaire (Gardin, 2006) comme une « hybridation entre accords formels et informels » (Nyssens, 1996).

\section{Cohérence de l'engagement économique, social et environnemental}

22 Afin de distinguer les entreprises sociales des administrations publiques, l'un des indicateurs de l'entreprise sociale d'EMES porte sur le niveau de prise de risque des créateurs de cette entreprise. Ce critère, qui mesure l'engagement économique des créateurs de l'entreprise, peut être étendu en incluant l'engagement social et environnemental.

23 Le consensus minimal sur l'agenda du développement durable, depuis le rapport Brundtland (1987) jusqu'aux Sommets de la terre de 1992, 2002 et 2012, s'est fait sur la reconnaissance de l'urgence d'intégrer les dimensions économiques, sociales et 
environnementales des activités humaines. Il semble dès lors difficile d'envisager que les entreprises sociales puissent, à la limite, s'engager dans des activités éventuellement risquées économiquement et nuisibles d'un point de vue social ou environnemental pour ensuite réinvestir le profit dégagé dans leur mission sociale.

Les fondateurs des entreprises solidaires, et, plus largement sans doute, de nombreux créateurs d'entreprises sociales, visent avant tout une cohérence de leur action dans les différents domaines y compris dans des entreprises solidaires informelles. La réflexion sur l'impact social et écologique de l'activité trouve sa place et génère des réponses concrètes. Par exemple une préférence est accordée aux intrants naturels ou à l'agroécologie, quand bien même cela augmente dans un premier temps les coûts de production (dans un second temps, ils sont valorisés sur des marchés spécifiques). En soumettant ces choix à des espaces internes de discussion démocratique, ces entreprises peuvent d'ailleurs développer des modes de priorisation des objectifs économiques, sociaux et environnementaux au niveau local. Ils offrent alors, par la constitution d'espaces publics de proximité (Eme, Laville, 2006) ou de micro-espaces publics autonomes, une réponse pratique à une préoccupation centrale d'écologistes critiques qui est l'insuffisance du paradigme de l'intégration des différents domaines lorsqu'il ne s'accompagne pas de modalités d'arbitrage concrètes (van Griethuysen, 2010).

\section{Valorisation du travail}

L'idéal-type d'EMES identifie un niveau « minimum » d'emploi rémunéré comme dernier critère économique des entreprises sociales. Ici aussi, l'expérience des entreprises solidaires, notamment celles issues de l'économie populaire, engage à aller plus loin en posant la valorisation du travail comme principe commun.

L'économie populaire a été définie comme "l'ensemble des activités économiques et pratiques sociales développées par les groupes populaires en vue de garantir par l'utilisation de leur propre force de travail et des ressources disponibles, la satisfaction des besoins de base, matériels autant qu'immatériels » (Sarria Icaza, Tiriba 2006, p. 259). Cette approche a le mérite de redécouvrir ces formes d'organisation populaire ayant une composante économique encastrée dans des relations sociales et culturelles invalidées depuis le XIX ${ }^{\mathrm{e}}$ siècle en étant présentées comme archaïques, dépassées et condamnées à disparaître avec la modernisation. Cette économie populaire ne peut être conceptualisée dans une simple dépendance à l'économie formelle, complément obligé d'un capitalisme sauvage ou expression volontaire d'un capitalisme « aux pieds nus » (De Soto, 1997). Pour José Luis Coraggio (2002, 2006), l'économie populaire est une économie du travail, en opposition à l'économie du capital parce qu'elle est mise en œuvre à partir de la logique du travail et de la reproduction de la vie au sein de l'unité domestique.

27 L'entreprise solidaire, ancrée dans cette économie populaire du travail, se positionne à l'encontre de la division sociale entre capital et travail. Elle privilégie les relations de travail entre associés et limite le recours à des travailleurs salariés qui ne sont pas membres de l'organisation. Elle tend à gérer l'organisation du travail et à déterminer sa rémunération selon des modes de décision démocratiques, indépendamment de la détention du capital, comme l'illustrent notamment les références à l'autogestion et à la coopérative. 


\section{Indicateurs sociaux}

\section{Finalité de transformation et réparation}

la communauté ou à un groupe de bénéficiaires, peut être spécifiée et problématisée dans le cas des entreprises solidaires à travers une double finalité de transformation et de réparation. Portées par la volonté d'aller vers un autre monde plus égalitaire et de résoudre des urgences, les entreprises solidaires sont à la fois transformatrices et réparatrices. La " logique contestataire (contester les règles et les valeurs en vigueur) » en leur sein ne peut être isolée d'« une logique palliative (améliorer l'existant) » (Blanc, Fare, 2012, p. 76). Cette ambivalence tient à l'importance pour leurs promoteurs de démocratiser l'économie par des engagements citoyens, partant du constat que la place excessive prise par un capitalisme patrimonial et financiarisé est aujourd'hui une cause essentielle du chômage, de la précarité et de la pauvreté.

souhait de transformation à long terme articulé à un pragmatisme de court terme débouche sur une conception du changement social qui ne se revendique pas «d'une nouvelle totalité, en rupture avec les déterminations actuelles ", mais qui est soucieuse d'apporter «une amélioration des conditions de vie» (Gaiger, 2006, p. 350-353). Cette attention aux conséquences humaines du changement évoque l'altermondialisme pour qui l'autre monde à inventer est déjà présent dans celui-ci. Son mot d'ordre "résister et construire » transposé sur le plan économique suppose de ne pas se prévaloir d'un autre système global, mais plutôt d'ancrer la perspective d'une autre économie dans les pratiques populaires et «d'inventer des alternatives (au pluriel)» (Sousa Santos et Rodriguez, 2013, 129). Cette perspective est congruente avec celle de l'innovation sociale et de la création de valeur sociale, à condition de tenir compte du type de processus (participatif ou non) et de finalité (transformatrice ou non) de l'innovation (Ferrarini, 2013).

\section{Solidarité démocratique au sein de l'entreprise solidaire}

Dans cette perspective, la vision de transformation et de réparation dans les entreprises solidaires ne peut pas être prise en compte uniquement par des mécanismes de limitation de la distribution des bénéfices. De manière plus large, c'est la volonté de démocratisation exprimée en pratique par des solidarités construites depuis la base qui entretient et légitime la finalité de ces entreprises :

- Pour ce qui est des formes adoptées, la solidarité n'est pas une solidarité traditionnelle dans laquelle les appartenances héritées peuvent conforter les hiérarchies fondées sur l'âge ou le sexe. Elle se démarque aussi d'une solidarité philanthropique renvoyant à la vision d'une société éthique où les citoyens motivés par l'altruisme remplissent leurs devoirs à l'égard des plus défavorisés sur une base volontaire.

- Pour ce qui est des personnes concernées, c'est une solidarité à la fois horizontale, qui vise le rééquilibrage des rapports entre les groupes sociaux actuellement vivants, et verticale, qui inclut les générations à venir. Il y a donc une volonté de lutte contre les inégalités et pour la justice sociale intégrant les aspects environnementaux.

31 L'entreprise solidaire s'appuie ainsi sur une solidarité que l'on peut qualifier de démocratique au sens où elle part du postulat d'égalité affirmé dans l'ordre politique 
pour le transposer dans la vie sociale et économique. Corollaire, la lutte contre la pauvreté n'est pas privilégiée contrairement à ce qui est le cas dans d'autres configurations de l'entreprise sociale. Il ne s'agit pas de faire preuve de compassion et de bienveillance pour des bénéficiaires, comme dans les "charities». Il s'agit que les personnes affectées par un problème puissent prendre en charge sa résolution, comme dans les « friendly societies », le « self-help » ou le « community development ». Le critère est celui de la participation active des bénéficiaires à la définition et la mise en œuvre de la mission de l'entreprise.

Ainsi, la protection obtenue par l'action collective vaut parce qu'elle est vectrice d'émancipation, c'est-à-dire de réalisation de soi. L'analyse de Nancy Fraser (2013) permet de bien expliciter cette articulation singulière. Partant de Polanyi qui a insisté sur les effets dévastateurs du « tout marché » et a montré comment la société se protégeait face à ce danger, Fraser note avec pertinence que la protection peut favoriser la domination ou au contraire l'émancipation. Elle complexifie le double mouvement (marchandisationprotection) de Polanyi pour le convertir en un triple mouvement (marchandisationprotection-émancipation). Dans ce cadre théorique, toute entreprise sociale aménage des formes de protection. L'entreprise solidaire, quant à elle, cherche à concilier protection et émancipation. La perspective solidaire insiste sur l'importance de l'émancipation et sur la mise œuvre d'actions qui articulent protection et émancipation, plutôt que de choisir entre l'une ou l'autre.

\section{Autonomie}

Finalement, le principe de structuration retenu est celui de l'auto-organisation à travers des logiques instituantes qui mixent dynamiques d'entraide et de transformation sociale. L'autogestion revendiquée dans le fonctionnement interne est indissociable de l'autonomie dans la prise de décision et dans l'accès aux connaissances stratégiques. Si les entreprises solidaires entretiennent des relations avec d'autres organisations et reçoivent des ressources pour l'accès aux marchés, à des financements ou à des connaissances techniques ou de gestion, cette aide ne doit pas entrainer la perte de contrôle de l'organisation. Les entreprises solidaires ne doivent pas non plus devenir de simples exécutants de programmes publics ou de projets sociaux de fondations privées. Comme le soulignent Jacques Defourny et Marthe Nyssens, les entreprises sociales sont « créées par un groupe de personnes sur base d'un projet propre et elles sont contrôlées par ces personnes (...). Elles ont le droit tant de faire entendre leur voix (voice) que de mettre un terme à leurs activités (exit) » (Defourny et Nyssens, 2013, p. 7).

\section{Indicateurs politiques}

En cohérence avec ce qui vient d'être énoncé, l'un des traits marquants de l'entreprise solidaire est de ne pas se considérer comme une organisation privée, mais de mener une action relevant du domaine public. Des critères d'ordre politique apparaissent donc indispensables pour caractériser les entreprises solidaires au-delà des indicateurs de structures de gouvernance de l'idéal-type d'EMES. 


\section{Dimension publique}

L'entreprise solidaire n'a pas qu'un rôle économique. Elle participe de la formulation de problèmes publics selon une approche du politique qui reconnait la place d'espaces publics dans lesquels peuvent être abordées les questions relevant du commun à partir de l'expression des citoyens. Les besoins sociaux ne sont pas repérés à partir de démarches marketing comme celles prônées pour le «bas de la pyramide » (Prahalad, 2004); comme mentionné plus haut, ils sont appréhendés grâce à des micro-espaces publics autonomes dans lesquels la délibération permet la redéfinition des intérêts et valeurs des participants (Hillenkamp, Bessis, 2012, p. 93). Cette élaboration itérative par contacts directs et échanges argumentaires est particulièrement pertinente dans les situations où les informations détenues par les protagonistes sont radicalement incomplètes et où c'est la structuration sociale de la situation qui autorise l'élaboration d'activités validées collectivement (Nyssens, 2006, p. 626). De tels espaces publics autonomes, constitués sur la base de la proximité, caractérisent la dynamique participative des entreprises solidaires, laquelle dépasse l'égalité juridique entre les membres. Son maintien dans la durée exige une vigilance particulière face à la menace d'isomorphisme institutionnel qu'engendrent les pressions de l'activité économique, comme en atteste l'abandon dans le passé de l'horizon d'autogestion (Singer, 2006, p. 294).

\section{Espaces publics intermédiaires}

La pérennité des micro-espaces publics autonomes existant au niveau des expériences singulières est envisageable seulement si des changements dans le cadre institutionnel permettent de s'attaquer aux discriminations négatives dont elles sont l'objet. Des regroupements territoriaux et sectoriels, la constitution de forums et d'arènes plus larges, s'avèrent donc indispensables pour impulser de telles évolutions à travers des espaces publics de niveau intermédiaire. Les institutions de médiation avec les pouvoirs publics, de représentation et de soutien sont d'autant plus utiles pour les entreprises solidaires qu'elles génèrent des apprentissages et donnent des résultats matériels et des gains extraéconomiques (França Filho et Laville, Gaiger 2006, p. 345).

La dynamisation de micro-espaces publics autonomes et d'espaces publics intermédiaires est essentielle pour des stratégies économiques non capitalistes, prises en charge par les acteurs de la société civile face aux dérives de l'économie dominante. Ces espaces publics sont décisifs pour une relance de l'implication citoyenne qu'ils induisent et que la démocratie représentative ne suffit pas à obtenir.

\section{Entrepreneuriat institutionnel et encastrement politique}

Ils le sont aussi parce qu'il serait naïf de croire que les entreprises solidaires s'imposent par leurs performances économiques. Elles pâtissent constamment de discriminations négatives inscrites dans le cadre institutionnel au sens large. Un changement à ce niveau est indispensable, principalement dans les cadres légaux et dans les politiques publiques, qui sont à déconstruire (en délégitimant les pouvoirs et hiérarchies institués) et à construire (en reconnaissant des activités auparavant ignorées). Cette activité en faveur d'un changement institutionnel a été désignée comme "entrepreneuriat institutionnel » (Lawrence, Suddaby 2006) et son importance a été soulignée par l'UNESCO stipulant dans sa déclaration universelle sur la diversité culturelle de 2001 que « face à la concentration 
oligopolistique les États doivent associer étroitement les différents secteurs de la société civile ». Alors que la littérature sur l'entrepreneuriat institutionnel s'est intéressée aux organisations dans leur ensemble, des cas étudiés dans le secteur médical (Lévy et Scully, 2007) et environnemental (Quéinnec, 2007) soulignent le rôle des associations (Rival et al. 2008). Dans cette lignée les entreprises solidaires constituent certainement l'un des domaines à approfondir.

Il devient ainsi crucial d'analyser l'encastrement politique, c'est-à-dire les interactions entre initiatives de la société civile se référant à un bien commun et les législations et politiques publiques. Les entreprises solidaires, comme d'autres initiatives, peuvent influer sur les modes d'action publique en même temps qu'elles sont normalisées par les pouvoirs publics, par le biais de processus d'institutionnalisation qui ne sont ni pure reproduction ni pure innovation.

Tableau. L'idéal-type d'entreprise sociale

dans une perspective d'économie solidaire

\begin{tabular}{l|l}
\hline Indicateurs & Entreprise solidaire \\
\hline Économiques & $\begin{array}{l}\text { Hybridation des principes économiques et logiques de solidarité } \\
\text { Cohérence de l'engagement économique, social et environnemental } \\
\text { Valorisation du travail }\end{array}$ \\
\hline Sociaux & $\begin{array}{l}\text { Finalité de transformation et réparation } \\
\text { Solidarité démocratique } \\
\text { Autonomie }\end{array}$ \\
\hline Politiques & $\begin{array}{l}\text { Dimension publique } \\
\text { Espaces publics intermédiaires } \\
\text { Entrepreneuriat institutionnel et encastrement politique }\end{array}$ \\
\hline
\end{tabular}

\section{Conclusion}

Les indicateurs de l'entreprise solidaire synthétisent des comportements stylisés. En tant qu'idéal-type, « le concept d'entreprise solidaire est un instrument heuristique, utile dans la recherche des connexions causales, non accidentelles, qui sont à l'œuvre au sein des expériences d'économie solidaire et les constituent en tant que catégorie spécifique d'initiatives économiques » (Gaiger, 2006, p. 355).

41 Sa mise en perspective avec les autres approches de l'entreprise sociale permet de plus un retour réflexif sur celles-ci. Les critères dégagés montrent en effet la dimension à la fois sociale, économique et politique des entreprises solidaires, liées entre elles par un noyau normatif explicite. Par contraste, la normativité implicite dans les courants américains des ressources marchandes et de l'innovation sociale apparait clairement.

En effet, le courant des ressources marchandes avalise sur le plan théorique ce que Polanyi (2007) nomme un "sophisme économiste", soit une confusion lancinante entre économie et marché. L'augmentation des ressources marchandes y est appréciée positivement, sans questionner les effets induits, selon une approche formelle de l'économie qui, contrairement à l'approche substantive, ne reconnait pas la pluralité des principes économiques. Pourtant, sur le plan empirique, le formalisme et cette focalisation sur les ressources marchandes ont déjà produit des effets pervers: le 
microcrédit illustre comment la recherche d'un autofinancement par le marché a entrainé un écrémage des populations touchées et un risque de surendettement (Guérin, Morvant-Roux et Villareal 2013). Cette propension à faire d'un simple outil un objet de croyance, symptomatique dans le microcrédit, devrait inciter à la perplexité devant l'héroïsation des personnalités d'entrepreneurs sociaux qui auraient «la capacité à transformer le monde» (Bornstein, 2004) en propageant un "social business» fonctionnant, selon Mohammad Yunus (2008), « conformément aux principes de gestion qui ont cours dans une entreprise classique » et ayant vocation à " couvrir complètement ses coûts ». Une telle littérature sur l'entreprise sociale imbrique dimensions analytique et promotionnelle, ce qui peut conduire à un isomorphisme marchand en psychologisant l'entrepreneuriat social ou en recommandant l'importation des méthodes managériales privées.

Dans ce contexte, l'idéal-type d'entreprise solidaire, par son registre plus institutionnel et politique, élargit la gamme des déclinaisons possibles de l'entreprise sociale, enrichissant les débats qui gagneraient à inclure des perspectives critiques extérieures au monde anglophone comme par exemple celles de Michel Foucault (2004) dénonçant le mouvement qui fait de l'entreprise la seule forme d'action collective légitime ou de Christian Laval (2007, p. 333) étudiant comment l'entreprise est actuellement présentée " comme une forme universelle d'action ».

Par la pluridimensionnalité de ses critères, par l'attention portée à la gouvernance interne (Eynaud, 2015), l'entreprise solidaire se rapproche indéniablement de l'idéal-type identifié par le réseau européen EMES, avec toutefois des traits comme la finalité de transformation et de réparation, la pluralité des principes économiques et la dimension publique qui témoignent de réalités observables notamment dans d'autres contextes. Il n'est pas concevable d'ignorer celles-ci dans la théorisation en devenir de l'entreprise sociale et l'idéal-type présenté dans cette contribution entend intégrer la réflexion sur ces pratiques dans une dimension ouverte et pluriculturelle. Il a pour objet de constituer un idéal-type qui puisse être mobilisé en dialogue et en complément avec celui déjà établi dans les travaux du réseau EMES.

\section{BIBLIOGRAPHIE}

H. Arendt, Condition de l'homme moderne, Paris, Calmann-Lévy, 1983

J. E Austin, B. Leonard, E. Reficco et J. Wei-Skillern, « Social Entrepreneurship : It's for Corporations too ", in Nicholls, A. (dir.) Social Entrerpreneurship, New Models of Sustainable Social Change, Oxford, Oxford University Press, 2006, pp. 169-80

D. Bornstein, How to change the World: Social Enterpreneurs and the Power of New Ideas, New York, Oxford University Press, 2004

J. Blanc, M. Fare, "Les monnaies sociales en tant que dispositifs innovants : une évaluation », in Innovations sociales $n^{\circ} 38,2012$

F. Braudel, La dynamique du capitalisme, Paris, Arthaud, 1985 
M. de Certeau, L'invention du quotidien, Paris, Gallimard, 1980.

Co-operative and Policy Alternative Center (COPAC), Beyond the Social Economy. Capitalism's crises and the Solidarity Economy Alternative, Conference Report, University of Witwatersrand, Johannesburg, Afrique du Sud, 2011.

J.L. Coraggio, La gente o el capital. Desarollo local y economia del trabajo, Buenos Aires, Espacio editorial, 2002

J.L. Coraggio, « Economie du travail », in J-L. Laville, A.D. Cattani, Dictionnaire de l'autre économie, Paris, Gallimard Folio, 2006

J-G. Dees, « Enterprising Nonprofits », Harvard Business review, January-February, 1998

J. Defourny et M. Nyssens, «L'approche EMES de l'entreprise sociale dans une perspective comparative », SOCENT Working Paper 2013/01, in partnership with EMES network, 2013

J. Defourny, M. Nyssens, «Les conceptualisations internationales de l'entreprise sociale », in L. Gardin, J-L. Laville, M. Nyssens, Entreprise sociale et insertion, Paris, Desclée De Brouwer, 2013

H. De Soto, El otro sendero, Traduction française : L'autre sentier. La révolution informelle, Paris, La Découverte, 1997

B. Eme et J.-L. Laville, «Économie solidaire (2) », in J.L. Laville et A.D. Cattani (dir.), Dictionnaire de l'autre économie, Paris, Gallimard, 2006

P. Eynaud, La gouvernance entre diversité et normalisation, Dalloz, Juris éditions, Hors série Vie associative, 2015

A.V. Ferrarini, o ethos da inovação social no ambiente das lutas populares e da economia solidária. Anais do XVI Congresso Brasileiro de Sociologia, Salvador, 2013

B. Fonteneau et al.,Economía Social y Solidaria : nuestro camino común hacia el trabajo decente. Turin : Centre international de formation de l'OIT, 2011

G.C. França Filho , « Políticas públicas de economia solidária no Brasil : características, desafios e vocação ", in França Filho Genauto Carvalho, Jean-Louis Laville, et al. (dir.), Ação Pública e economia solidária - uma perspectiva internacional, Salvador, Porto Alegre, EDUFRGS/EDUFBA, pp. 259-67, 2006

N. Fraser, « Marchandisation, protection sociale, émancipation : vers une conception néopolanyienne de la crise capitaliste », in Hillenkamp Isabelle et Jean-Louis Laville (dir.), Socioéconomie et démocratie. L'actualité de Karl Polanyi, Toulouse, Erès, pp. 39-63, 2013

M. Foucault,, Naissance de la biopolitique, Cours au collège de France 1978-1979, Paris, GallimardSeuil, 2004

L.I. Gaiger, « Entreprise solidaire », in J-L. Laville, A.D. Cattani, Dictionnaire de l'autre économie, Paris, Gallimard Folio, 2006

L.I Gaiger, «O Mapeamento Nacional e o Conhecimento da Economia Solidária », Revista da ABET (En ligne) $\mathrm{n}^{\circ}$ 12, pp. 7-24, 2013.

L. Gardin,Les initiatives solidaires : la réciprocité face au marché et à l'Etat. Ramonville Saint-Agne : Erès, 2006.

L. Gardin, J.L. Laville, M. Nyssens (2012). Entreprise sociale et insertion : une perspective internationale, Paris, Desclée de Brouwer, 2012

I. Guérin, S. Morvant-Roux, M. Villarreal (dir.), Microfinance, Debt and Over-indebtedness : Juggling with Money, Routledge, 2013 
J. Habermas, Théorie de l'agir communicationnel, Paris, Fayard, 1998

J. Habermas, L'espace public. Archéologie de la publicité comme dimension constitutive de la société bourgeoise, Paris, Payot, 1988

I. Hillenkamp, F. Bessis, «L'innovation sociale par l'économie solidaire en Bolivie : une démocratisation des conventions de production et de genre », in Innovations sociales $n^{\circ} 38,2012$

I. Hillenkamp, « Le principe de householding aujourd'hui. Discussion théorique et approche empirique par l'économie populaire », in Hillenkamp Isabelle et Jean-Louis Laville (dir.), Socioéconomie et démocratie. L'actualité de Karl Polanyi, Toulouse, Erès, pp. 215-39, 2013

C. Laval, L'homme économique. Essai sur les racines du néolibéralisme, Paris, Gallimard, 2007

B. Lautier, « Economie informelle », in J-L. Laville, A.D. Cattani, Dictionnaire de l'autre économie, Paris, Gallimard Folio, 2006

T-B. Lawrence, R. Suddaby, « Institutions and Institutional Work », S.R. Clegg (ed.), Handbook of organization studies, (2 éd.), Londres, Sage, 2006

D. Lévy, M. Scully, “The institutional entrepreneur as modern Prince : the strategic fact of power in contested fields", Organization Studies, 28(07), p. 971-991

M. de Nanteuil, J-L. Laville, «Crise du capitalisme et économie plurielle : une perspective anthropologique », Revue Option, Bruxelles, Confrontation Europe, 2013

M. Nyssens, 'Popular Economy in the South, Third Sector in the North : Seeds of a Mutually Supportive Sector ?', in P. Sauvage (ed) Reconciling Economy and Society. Towards a Plural Economy, pp. 91-115, Paris : OECD, 1996

M. Nyssens, Social Enterprise at the crossroads of Market, Public Policies and Civil - Society, London and New York, Routledge Taylor \& Francis Group, 2006

E. Ostrom, Understanding Institutional Diversity, Princeton/Oxford, Princeton University Press, 2005.

R. Paton, Relunctant Entrepreneurs. The Extent, Achievements ans Significance of Worker Takeovers in Europe, Milton-Keynes, Philadelphia, Open University Press, 1989

K. Polanyi, « Le sophisme économiciste », Revue du MAUSS semestrielle, Avec K. Polanyi, contre la société du tout marchand, $n^{\circ} 29,2007$

K. Polanyi, «L'économie en tant que procès institutionnalisé », in Polanyi Karl, Conrad M. Arensberg et Harry Pearson (dir.), Les systèmes économiques dans l'histoire et dans la théorie, Paris, Larousse université, pp. 239-60, 1975 [1957]

K. Polanyi, La subsistance de l'homme. La place de l'économie dans l'histoire et la société, (traduit et présenté par B. Chavance), Paris, Flammarion, 2011

C.K. Prahalad, Fortune at the bottom of the pyramid, ED. Village Mondial, 2004

E. Quéinnec, «La croissance des ONG humanitaires, une innovation devenue institution ", Revue française de gestion, 33 (117), p. 83-94

L. Razeto, Empresas de trabajadores y economia de mercado, Santiago, Chili, Programa de Economia del Trabajo, Academia de Humanismo Christiano, 1993

M. Rival, P. Eynaud, A. Gautier. « Associations et entrepreneuriat institutionnel », in C. Hoarau, JL. Laville (dir.), La gouvernance des associations, Toulouse, éditions Eres, 2013, p. 264-277 
M-D. Salamon, D.Young, The State of Nonprofit America, Washington DC, Brookings Institution, 2002

A-M Sarria Icaza., L. Tiriba, (2006), « Économie populaire », in Laville Jean-Louis et Cattani Antonio David (dir.), Dictionnaire de l'autre économie, Paris, Gallimard, pp. 258-68.

J-M. Servet,., Banquiers aux pieds nus. La microfinance, Paris, Odile Jacob, 2006

J-M. Servet., « Le principe de réciprocité aujourd'hui. Un concept pour comprendre et construire l'économie solidaire ", in Hillenkamp Isabelle et Jean-Louis Laville (dir.), Socioéconomie et démocratie. L'actualité de Karl Polanyi, Toulouse, Erès, pp. 187-213, 2013

B. Sousa Santos, C. Rodríguez Garavito, « Alternatives économiques : les nouveaux chemins de la contestation », in Hillenkamp Isabelle et Jean-Louis Laville (dir.), Socioéconomie et démocratie. L'actualité de Karl Polanyi, Toulouse, Erès, pp. 127-47, 2013

P. Singer, «Economie solidaire », in J-L. Laville, A.D. Cattani, Dictionnaire de l'autre économie, Paris, Gallimard Folio, 2006

P. Van Griethuysen, « Pourquoi le développement durable s'est-il imposé là où

l'écodéveloppement a échoué ? Débats pour la suite du monde », in Abraham Yves-Marie, Marion Louis et Philippe Hervé (dir.), Développement durable ou décroissance soutenable?, Montréal, Ecosociété, pp. 60-79, 2010

F. Wanderley, Crecimiento, empleo y bienestar social ¿Por qué Bolivia es tan desigual ?, La Paz, Plural, 2009

M. Yunus, Vers un nouveau capitalisme, Paris, Jean-Claude Lattes, 2008

\section{NOTES}

1. Le réseau EMES se positionne : économie sociale, économie solidaire, entreprise sociale, entrepreneuriat social, innovation sociale. http://emes.net

2. Defourny Jacques et Nyssens Marthe (2013), L'approche EMES de l'entreprise sociale dans une perspective comparative, SOCENT Working Paper 2013/01, in partnership with EMES network. 